\title{
A Novel Penalty-Based Reduced Order Modelling Method for Dynamic Analysis of Joint Structures
}

Jie Yuan, Loic Salles, Chian Wong, Sophoclis Patsias

\begin{abstract}
This work proposes a new reduced order modelling method to improve the computational efficiency for the dynamic simulation of a jointed structures with localized contact friction non-linearities. We reformulate the traditional equation of motion for a joint structure by linearising the non-linear system on the contact interface and augmenting the linearised system by introducing an internal non-linear penalty variable. The internal variable is used to compensate the possible non-linear effects from the contact interface. Three types of reduced basis are selected for the Galerkin projection, namely, the vibration modes (VMs) of the linearised system, static modes (SMs) and also the trial vector derivatives (TVDs) vectors. Using these reduced basis, it would allow the size of the internal variable to change correspondingly with the number of active non-linear DOFs. The size of the new reduced order model therefore can be automatically updated depending on the contact condition during the simulations. This would reduce significantly the model size when most of the contact nodes are in a stuck condition, which is actually often the case when a jointed structure vibrates. A case study using a 2D joint beam model is carried out to demonstrate the concept of the proposed method. The initial results from this case study is then discussed.
\end{abstract}

Jie Yuan

Vibration University Technology Centre, Department of Mechanical Engineering, Imperial College London e-mail: Jie.Yuan@imperial.ac.uk

Loic Salles

Vibration University Technology Centre, Department of Mechanical Engineering, Imperial College London e-mail: L.Salles@imperial.ac.uk

Chian Wong

Rolls Royce plc e-mail: Chian.Wong@Rolls-Royce.com

Sophoclis Patsias

Rolls Royce plc e-mail: sophoclis.patsias@rolls-royce.com 


\section{Introduction}

Jointed structures have been widely used in gas turbine engine to transfer the loading from one component to the other. The joints between the substructures such as the shrouds, underplatform damper and dovetail joint in gas turbine engines are also regarded as the primary damping sources for energy dissipation. However, they also significantly complicate the dynamic behaviour of an assembly by the change of stability, the jump phenomenon and energy localization [4]. It is therefore very important to understand and improve the dynamics of the joints for design. The use of finite element (FE) method for analysing such dynamical systems is however often impeded by the unacceptable computational expense due to the tremendous size of the model and strong inherit contact friction nonlinearities. The harmonic balanced method (HBM) provides a very efficient approach to obtain the steady state dynamic behaviour of such jointed structures comparing to the time integration method [4]. However, HBM would expand the size of the orgianl system by multiplying the chosen number of harmonic coefficients [6]. One of the viable approaches to take these nonlinearities into account is to reduce the model size by several orders of magnitudes by employing reduced order modelling (ROM) techniques [4, 6, 12].

Component mode synthesis (CMS) techniques have been extensively used for model order reduction for linear and locaised nonlinear dynamic systems where the physical nonlinear DOFs on the interface are retained as unkowns [8, 13]. A CMS-Hybrid approach based on free interface modes and flexible residual have been successfully applied to the Imperial VUTC in-house FORSE solver [6]. Another effective CMS approach is on the use of Craig-Bampton (CB) method [2]. A review of CMS based ROM techniques for the applocations to the linear vibration and locaized nonlinear vibration can be referred to $[3,12]$. The main drawback of these two approaches is the size of the reduced model is proportional to the number of DOFs involved in nonlinearities. It could become extremely large when nonlinear interface regions are intentensive and densely meshed $[10,12]$. In terms of modeling contact friction on the interface, a node-to-node modeling approach has been widely used [1] and also experimentally validated for turbine underplatform damper at Imperial VUTC [5]. The contact friction conditions can be described as in stuck, slip and gap states. More details on the contact friction modelling would be introduced in Sec. 2. Fig. 1 shows the forced frequency response of a turbine blade and also the average contact conditions of the interface nodes during the non-linear dynamic analysis. An interesting observation is that most of the contact nodes are actually in a stuck condition under. When the contact interface is in a stuck condition, the coupling between two contact interfaces can be represented as the linear springs. An inspiration from this observation is that we can linearise the non-linear system using linear springs, and compensate the non-linear effect from those contact nodes in a slip and gap conditions with a internal penalty variable.

This paper aims to investigate this penalty-based ROM approach in order to further reduce the model size comparing to those reduced models using classical CMS methods. The paper is organized as follows: the equation of motion (EOM) of this penalty approach will be firstly presented; it is followed by the presentation of re- 

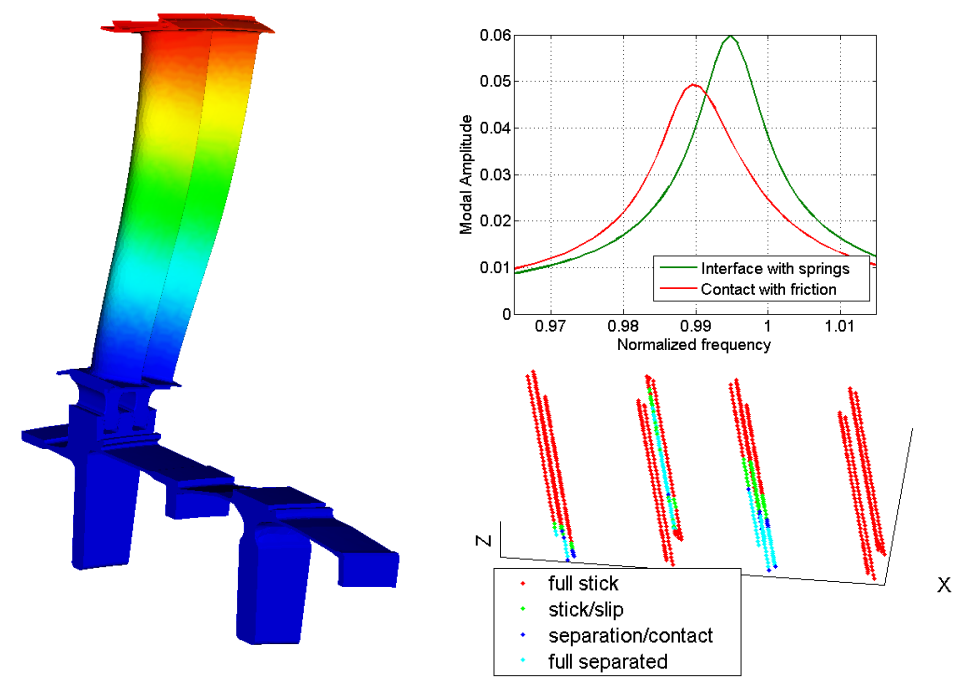

Fig. 1 An example of the contact conditions of a turbine during the vibrations

duced basis for Galerkin projection; we will then elaborate how this method can be coupled with the harmonic balanced method; the performance of this approach will be then demonstrated using a 2D jointed beam case study.

\section{Formulation}

\subsection{Equation of motion}

A dynamic system consisting of two connected substructures with localized contact friction interfaces is considered as an example. The partial differential governing equation of such a system without taking into account of damping matrix is:

$$
\mathbf{M} \ddot{u}+\mathbf{K} u=F-F_{n l}(u, \ddot{u})
$$

It is assumed the size of each substructures is $N$ and the size of contact DOFs for each substructure is $M$. Using the finite element modelling method, the assembled system with two substructures can be expressed as:

$$
\left[\begin{array}{cc}
\mathbf{M}_{1} & \mathbf{0} \\
\mathbf{0} & \mathbf{M}_{2}
\end{array}\right]\left[\begin{array}{l}
\ddot{u_{1}} \\
\ddot{u_{2}}
\end{array}\right]+\left[\begin{array}{cc}
\mathbf{K}_{\mathbf{1}} & \mathbf{0} \\
\mathbf{0} & \mathbf{K}_{2}
\end{array}\right]\left[\begin{array}{l}
u_{1} \\
u_{2}
\end{array}\right]=\left[\begin{array}{l}
F_{1} \\
F_{2}
\end{array}\right]-\left(\left[\begin{array}{l}
\mathbf{B}_{1}^{\mathbf{T}} \\
\mathbf{B}_{2}^{\mathbf{T}}
\end{array}\right] F^{T}\left(u_{1}, u_{2}\right)+\left[\begin{array}{l}
\mathbf{B}_{1}^{\mathbf{N}} \\
\mathbf{B}_{\mathbf{2}}^{\mathbf{N}}
\end{array}\right] F^{N}\left(u_{1}, u_{2}\right)\right)
$$

Where $\mathbf{M}_{1}, \mathbf{M}_{2}, \mathbf{K}_{1}, \mathbf{K}_{2}$ are the mass and stiffness matrix of two substructures with the same dimensions of $N x N ; F^{T}$ and $F^{N}$ are the $2 M x 1$ non-linear contact fric- 
tion force vector, which are in a function of the relative displacement of the contact interface in a joint structure. $\mathbf{B}^{\mathbf{T}}$ and $\mathbf{B}^{\mathbf{N}}$ are the $2 N x 2 M$ boolean matrix related to the boundary DOFs in tangential and normal direction separately.

\subsection{Contact friction modelling}

A node-to-node approach with Jenkin model is used to model the contact friction phenomenon in a joint, which includes stuck, slip and gap states. These contact friction states are dependent on the preloading levels as well as the amplitude of relative movements on the interface. The Jenkin model has been widely used and also validated with experiments [1]. The formulation for a 3D contact node pair using this Jenkin model can be written as:

$$
\begin{gathered}
F_{x}^{T}=\left\{\begin{array}{l}
k_{t}\left(\Delta x-\Delta x_{c}\right)+N_{x}^{0}, \text { stuck } \\
\mu F_{z}^{N} \sin (\theta), \text { slip } \\
0, \text { gap }
\end{array}\right. \\
F_{y}^{T}=\left\{\begin{array}{l}
k_{t}\left(\Delta y-\Delta y_{c}\right)+N_{y}^{0}, \text { stuck } \\
\mu F_{z}^{N} \cos (\theta), \text { slip } \\
0, \text { gap }
\end{array}\right. \\
F_{z}^{N}=\left\{\begin{array}{l}
k_{n} \Delta z+N_{z}^{0}, \text { stuck } \\
k_{n} \Delta z+N_{z}^{0}, \text { slip } \\
0, \text { gap }
\end{array}\right.
\end{gathered}
$$

Where $\Delta x, \Delta y$ and $\Delta z$ are the time-dependent tangential and normal relative displacement of a contact node pair; $\Delta x_{c}, \Delta y_{c}$ are internal variables representing the tangential position of the slider, which also needs to update at each time step; $F_{x}^{T}, F_{y}^{T}$ are the tangential force in $x$ and $y$ direction; $F_{z}^{N}$ is the normal force in $z$ direction; $\theta$ is the angle between the tangential force component in $x$ and $y$ direction, which is determined by the predicted tangential force:

$$
\theta=\arctan \left(\frac{k_{t}\left(\Delta x-\Delta x_{c}\right)+N_{x}^{0}}{k_{t}\left(\Delta y-\Delta y_{c}\right)+N_{y}^{0}}\right)
$$

The stuck condition occurs when the predicted tangential force $\sqrt{F_{x}^{T^{2}}+F_{y}^{T^{2}}}$ is less than the critical slipping force $\mu F_{z}^{N}$. The contact force would behave linearly and there would be no energy dissipation. The slip condition occurs when the predicted tangential friction force is larger than the critical value $\mu F_{z}^{N}$. The tangential contact force would behave non-linearly with a value of $\mu F_{z}^{N}$ and the energy dissipation would happen then. The gap condition would happen when predicted normal force $F_{z}^{N}$ is less than zero, and all the contact force would be zero then. 


\section{Penalty-based approach}

\subsection{EOM modification}

For the proposed penalty-based approach, the original EOM in Eq. 2 is linearised on the interface using contact stiffness by assuming all of the contact nodes are in a stuck condition. The modified linearised EOM of the system can be expressed as:

$$
\left[\begin{array}{cc}
\mathbf{M}_{1} & \mathbf{0} \\
\mathbf{0} & \mathbf{M}_{2}
\end{array}\right]\left[\begin{array}{l}
\ddot{u}_{1} \\
\ddot{u}_{2}
\end{array}\right]+\left[\begin{array}{cc}
\mathbf{K}_{1}+\mathbf{K}_{\mathbf{J o i n t}} & -\mathbf{K}_{\mathbf{J o i n t}} \\
-\mathbf{K}_{\mathbf{J o i n t}} & \mathbf{K}_{\mathbf{2}}+\mathbf{K}_{\mathbf{J o i n t}}
\end{array}\right]\left[\begin{array}{l}
u_{1} \\
u_{2}
\end{array}\right]=\left[\begin{array}{c}
F_{1} \\
F_{2}
\end{array}\right]
$$

Where $\mathbf{K}_{\text {Joint }}$ is the $N x N$ stiffness matrix containing the local stiffness matrix associated to the joint DOFs. The linearised stiffness matrix in Eq.7 is denoted as Klinearized.

When any contact nodes are in a slip or gap condition, an internal variable $\Delta p$ would be needed to augment linearised EOM. The dimension of $\Delta p$ is $M x 1$, which is the half number of the total joint DOFs. The internal variable would become zero when a contact node are in a stuck condition. The expression of the internal variable can be formulated as:

$$
\Delta p=\left\{\begin{array}{l}
0, \text { stuck } \\
\mathbf{K}_{\text {Joint }}^{\mathbf{n l , n l}-1}\left(F_{n l}(\Delta u)-\mathbf{K}_{\text {Joint }}^{\mathbf{n l}} \Delta u\right), \text { slip } \\
\mathbf{K}_{\text {Joint }}^{\mathbf{n l , n l}-1}\left(F_{n l}(\Delta u)-\mathbf{K}_{\text {Joint }}^{\mathbf{n l}} \Delta u\right), \text { gap }
\end{array}\right.
$$

Where $\mathbf{K}_{\text {Joint }}^{\mathbf{n l}, \mathbf{n l}}$ is the $M x M$ joint stiffness matrix associated to non-linear internal variable; $\mathbf{K}_{\text {Joint }}^{\text {nl }}$ is the $N x M$ joint matrix relating to the DOFs in each substructure. $\Delta u$ is the assembly of relative displacement $(\Delta x, \Delta y, \Delta z)$ of all the contact pairs in joint interfaces. By integrating the internal penalty variable, the modified EOM can be further augmented:

$$
\left[\begin{array}{ccc}
\mathbf{M}_{1} & \mathbf{0} & \mathbf{0} \\
\mathbf{0} & \mathbf{M}_{2} & \mathbf{0} \\
\mathbf{0} & \mathbf{0} & \mathbf{0}
\end{array}\right]\left[\begin{array}{c}
\ddot{u}_{1} \\
\ddot{u}_{2} \\
\Delta \ddot{p}
\end{array}\right]+\left[\begin{array}{ccc}
\mathbf{K}_{\mathbf{1}}+\mathbf{K}_{\mathbf{J o i n t}} & -\mathbf{K}_{\mathbf{J o i n t}} & \mathbf{K}_{\mathbf{J o i n t}}^{\mathbf{n l}} \\
-\mathbf{K}_{\mathbf{J o i n t}} & \mathbf{K}_{\mathbf{2}}+\mathbf{K}_{\mathbf{J o i n t}} & -\mathbf{K}_{\mathbf{J}}^{\mathbf{n l}} \\
\mathbf{K}_{\mathbf{J}^{\prime}}^{\mathbf{n}} & -\mathbf{K}_{\mathbf{J o i n t}^{\prime}} & \mathbf{K}_{\mathbf{J o i n t}}^{\mathbf{n l}, \mathbf{n l}}
\end{array}\right]\left[\begin{array}{c}
u_{1} \\
u_{2} \\
\Delta p
\end{array}\right]=\left[\begin{array}{c}
F_{1} \\
F_{2} \\
F_{n l}\left(u_{1}, u_{2}\right)
\end{array}\right]
$$

Here, $\mathbf{M}_{\mathbf{N e w}}, \mathbf{K}_{\text {New }}$ are used to denote as new assembled mass and stiffness matrix. It is worth noting that the zero part of $\Delta p$ associated to the contact nodes in a stuck condition can be further eliminated in EOM. The following section would detail how the ROM formulates to enable such an automatic updating. 


\subsection{Reduced basis}

Galerkin projection is used to reduce the size of a physical model by transforming it into a subspace. The solution of the system can be expressed as a linear combination of vectors spanning the subspace. The selection of the reduced basis is crucial in determining the accuracy and computational efficiency of a reduced system. More about Galerkin projection can be referred to [7, 12]. The reduced basis for the proposed penalty approach contain three parts, namely the vibrational modes of the corresponding linearised system, constrain (static) modes and also the modal derivatives vectors (Trial Vector Derivatives). The vibration modes can be obtained by solving the eigenvalue problem of the linearised system Eq.2:

$$
-\omega^{2}\left[\begin{array}{cc}
\mathbf{M}_{1} & \mathbf{0} \\
\mathbf{0} & \mathbf{M}_{2}
\end{array}\right][\phi]+\left[\begin{array}{cc}
\mathbf{K}_{1}+\mathbf{K}_{\text {Joint }} & -\mathbf{K}_{\text {Joint }} \\
-\mathbf{K}_{\text {Joint }} & \mathbf{K}_{2}+\mathbf{K}_{\text {Joint }}
\end{array}\right][\phi]=[0]
$$

Like CMS techniques, the static modes are used to approximate the high frequency response on the contact interface, which can be obtained by applying unit displacement vectors on the DOFs related to internal penalty variable.

$$
\left[\begin{array}{ccc}
\mathbf{K}_{\mathbf{1}}+\mathbf{K}_{\text {Joint }} & -\mathbf{K}_{\text {Joint }} & -\mathbf{K}_{\text {Joint }}^{\text {nl }} \\
-\mathbf{K}_{\text {Joint }} & \mathbf{K}_{\mathbf{2}}+\mathbf{K}_{\text {Joint }} & \mathbf{K}_{\text {Joint }}^{\text {Iloint }} \\
-\mathbf{K}_{\mathbf{J}^{\prime}}^{\mathbf{n}^{\prime}} & \mathbf{K}_{\mathbf{J o i n t}^{\prime}}^{\mathbf{l}^{\prime}} & \mathbf{K}_{\text {Joint }}^{\text {nl,nt }}
\end{array}\right]\left[\begin{array}{c}
\varphi \\
\mathbf{I}
\end{array}\right]=\left[\begin{array}{c}
\mathbf{0} \\
\mathbf{R}
\end{array}\right]
$$

Where $\mathbf{I}$ is the $M x M$ identity matrix; $\varphi$ is the $2 N x M$ matrix including all the static modes; $\mathbf{R}$ are the $M x M$ reaction force matrix.

TVDs are used to calibrate the linear reduced basis in order to consider the effect of the non-linearities from the contact friction. It is particularly usefully when all contact nodes are in a gap condition. The detailed formulation to calculate the TVDs can be referred to [11]. The first-order modal derivatives of the linear reduced basis can be calculated as follows:

$$
\varphi_{\mathbf{i}, \mathbf{j}}=\mathbf{K}_{\text {linearised }}^{-1} \frac{\partial \mathbf{K}_{\text {linearised }}}{\partial \mathbf{q}_{\mathbf{j}}} \varphi_{\mathbf{j}}, \phi_{\mathbf{i}, \mathbf{j}}=\mathbf{K}_{\text {linearised }}^{-1} \frac{\partial \mathbf{K}_{\text {linearised }}}{\partial \mathbf{q}_{\mathbf{j}}} \phi_{\mathbf{j}}
$$

Where $\varphi_{\mathbf{i}, \mathbf{j}}$ and $\phi_{\mathbf{i}, \mathbf{j}}$ are the TVDs from the linear vibration modes $\phi_{j}$ and constrain modes $\varphi_{j}$. The number of TVD vectors is equal to the squared number of the linear reduced basis. The proper orthogonal decomposition is then used to reduced the size of TVDs.

The transformation of the physical system to the modal domain can be shown as follows:

$$
\left[\begin{array}{l}
u_{1} \\
u_{2} \\
\Delta p
\end{array}\right]=\left[\begin{array}{ccc}
\phi & \phi_{\mathbf{T}} & \varphi \\
\mathbf{0} & \mathbf{0} & \mathbf{I}
\end{array}\right]\left[\begin{array}{l}
\eta_{1} \\
\eta_{2} \\
\Delta p
\end{array}\right]=\left[\begin{array}{ccc}
\phi & \phi_{\mathbf{T}} & \varphi \\
\mathbf{0} & \mathbf{0} & \mathbf{I}
\end{array}\right]\left[\begin{array}{lll}
\mathbf{I} & \mathbf{0} & \mathbf{0} \\
\mathbf{0} & \mathbf{I} & \mathbf{0} \\
\mathbf{0} & \mathbf{0} & \mathbf{B}
\end{array}\right]\left[\begin{array}{c}
\eta_{1} \\
\eta_{2} \\
\Delta p_{R}
\end{array}\right]
$$




$$
\mathbf{T}=\left[\begin{array}{ccc}
\phi & \phi_{\mathbf{T}} & \varphi \\
\mathbf{0} & \mathbf{0} & \mathbf{I}
\end{array}\right]\left[\begin{array}{lll}
\mathbf{I} & \mathbf{0} & \mathbf{0} \\
\mathbf{0} & \mathbf{I} & \mathbf{0} \\
\mathbf{0} & \mathbf{0} & \mathbf{B}
\end{array}\right], \mathbf{M}_{\mathbf{R}}=\mathbf{T}^{\prime} \mathbf{M}_{\mathrm{New}} \mathbf{T}, \mathbf{K}_{\mathbf{R}}=\mathbf{T}^{\prime} \mathbf{K}_{\mathrm{New}} \mathbf{T}
$$

where $\eta_{1}$ and $\eta_{2}$ are modal participation factors for vibration models and TVDs; $\Delta p_{R}$ is the non-zero part of $\Delta p ; \mathbf{B}$ is the index matrix to extract the non-zero part of $\Delta p$, which would be updated during the simulation depending on the contact conditions; the size of transformation matrix $\mathbf{T}$ would be also updated accordingly. As a result, the reduced mass and stiffness system $\mathbf{K}_{\mathbf{R}}$ and $\mathbf{M}_{\mathbf{R}}$ would be adaptively changing to reduce the computational time.

\section{Harmonic balanced method with continuation techniques}

Harmonic balanced method is used for solving the Eq.7. The idea of this method is to represent the steady state non-linear dynamic response using truncated Fourier series with $n$ harmonic series:

$$
u(t)=\tilde{u_{0}}+\sum_{i=1}^{n}\left(\tilde{u_{i}^{c}} \cos m_{i} \omega t+\tilde{u_{i}^{s}} \sin m_{i} \omega t\right)
$$

Where $u_{i}^{\tilde{c}, s}$ are cosine and sine harmonic coefficients; $\omega$ is the principal vibration frequency; $\tilde{u_{0}}$ is the zero harmonic response.The Newton-Raphson method, in coupling with the alternating frequency-time (AFT) method, is used to solve these nonlinear equations. The AFT technique is used to transforme the frequency-domain solution to the time domain for non-contact force calculation, and transforme nonlinear contact force back to frequency domain. More details about this part can be referred to [4]. Fig. 2 illustrates the implementation process about how the contact friction model,iterative Newton-Raphson solver work with reduced order modeling in HBM. The physical nonlinear DOFs $u_{b}$ on the contact interface is fristly expanded from modal subspace by $\mathbf{T}$ before employing the AFT procedure. After AFT procedure, the nonlinear force $F_{n l}$ is then projected back to the modal subspace. The continuation techniques are then used to obtain the forced frequency response. More details about continuation techniques can be referred to [9].

The automatic size updating depending on the contact condition is achieved as follows. When evaluating the continuous forced frequency response, the contact condition of all interface nodes would need to be assessed using the previous converged solution. This would help to generate the index matrix $\mathbf{B}$. The size of system can be then reduced for the new simulation. If using the continuation techniques with HBM, the size updating would be performed at the predictor stage. The size of the system would be kept same in the corrector stage with the iterative solver. The implementation of the size updating with HBM is still on-going research. 


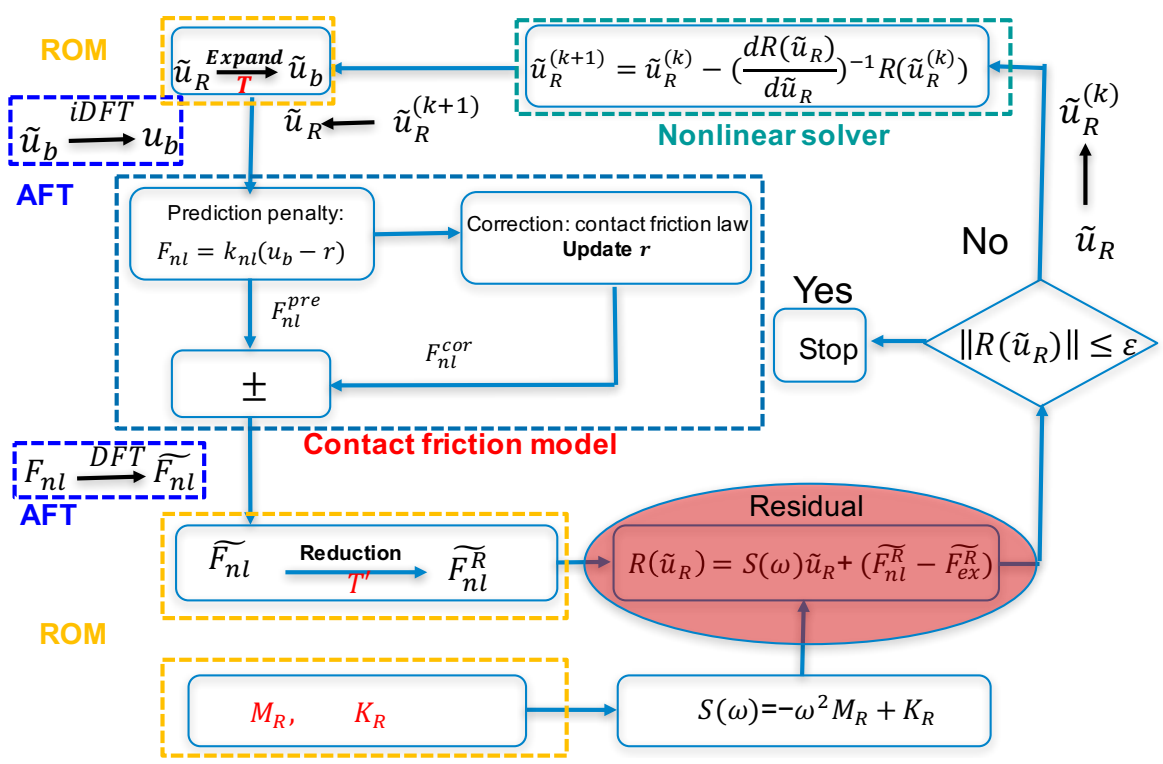

Fig. 2 An illustration of how Newton-Raphson solver works with AFT scheme and ROM methods

\section{Case study}

Fig. 3 shows a jointed beam model with linear springs connecting the two equivalent beam substructures. The length of each beam is $0.3 \mathrm{~m}$. The width and height of the cross section is $25 \mathrm{~mm}$ and $6 \mathrm{~mm}$ respectively. They are modelled by using the EulerBernoulli beam elements, where each node has three DOFs $\left(u_{x}, u_{y}, r_{z}\right)$. The beams are made of steel with a nominal density of $7850 \mathrm{~kg} / \mathrm{m} 3$ and Young's modulus of $2.1 e 11 N . m^{-2}$. The tangential stiffness of the springs in the joint is $1 e 4 N / m$ while normal contact stiffness is $5 e 6 \mathrm{~N} / \mathrm{m}$ and bending stiffness of $8 e 6 \mathrm{~N} . \mathrm{m} / \mathrm{rad}$.

Fig. 4 shows the first nine natural frequencies (NFs) and modes of this linearised jointed beam system. These nine modes all belong to the bending modes. Due to the large value of stiffness used in the joint, local elastic modes in the joint do not appear. The forced frequency response of the second bending mode would be studied.

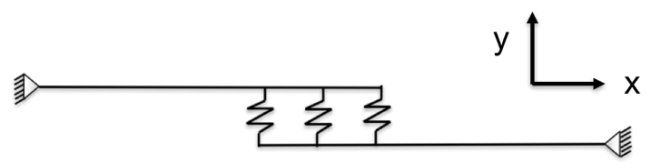

Fig. 3 A 2D FE model of a jointed beam with contact non-linear springs 

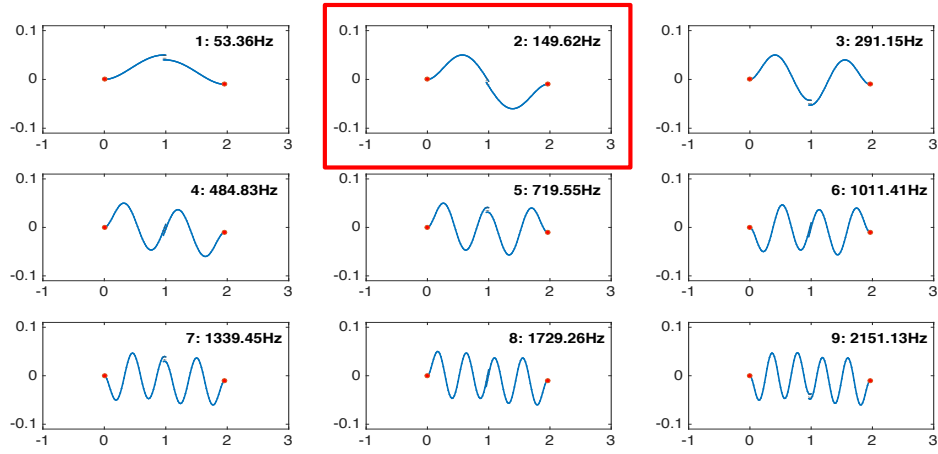

Fig. 4 Modes of a linearised jointed beam

The proposed method and also other reference methods are applied to the linearised joint beam model at first. The reference methods include CB, Rubin, Dual Craig-Bampton, MacNeal, Joint interface method and TVD methods. The formulation of these methods can be referred to [12]. The idea here is to compare the quality of these methods when all the contact nodes are in a stuck condition. Fig. 5 shows the comparison of the natural frequency (NF) errors between the proposed method and other reference methods. Except for the penalty method, all other methods have the same number of normal modes, namely 20 . In terms of the static modes, CB, Rubin and MacNeal methods have the same number as the non-linear DOFs while DCB and JIM methods have only half number of these non-linear DOFs. The static modes with TVD method is independent of the non-linear DOFs. In this case, the size of penalty method would be equal to the number of VMs in the linearised structure, because the number of static modes is zero due to the stuck condition. Fig. 5 shows the proposed method achieves the best accuracy with the smallest reduction ratio. RR stands for the reduction ratio, which is the ratio between the size of a ROM to the size of a full system.

For linear analysis, it is well known that the pre-processing effort would be the main challenge when using the CMS techniques, because the inversion of matrix during the dynamic analysis would be only needed once for each frequency. For non-linear analysis, the reduced size of system would be also a challenge especially when the assemble structure contains intensive contact interfaces. It is because the iterative solver would be needed to solve the reduced system until the solution converges. Furthermore, using the HBM, the final size of system would be expanded by the number of harmonics. Based on the authors' previous simulation, the computational time would be cubic relation to the reduced number of DOFs [5]. Therefore, the RR would be particularly important to non-linear vibration analysis. In terms of the off-line cost, the proposed method has the same computational time for preprocessing when comparing to the classical CMS techniques. 


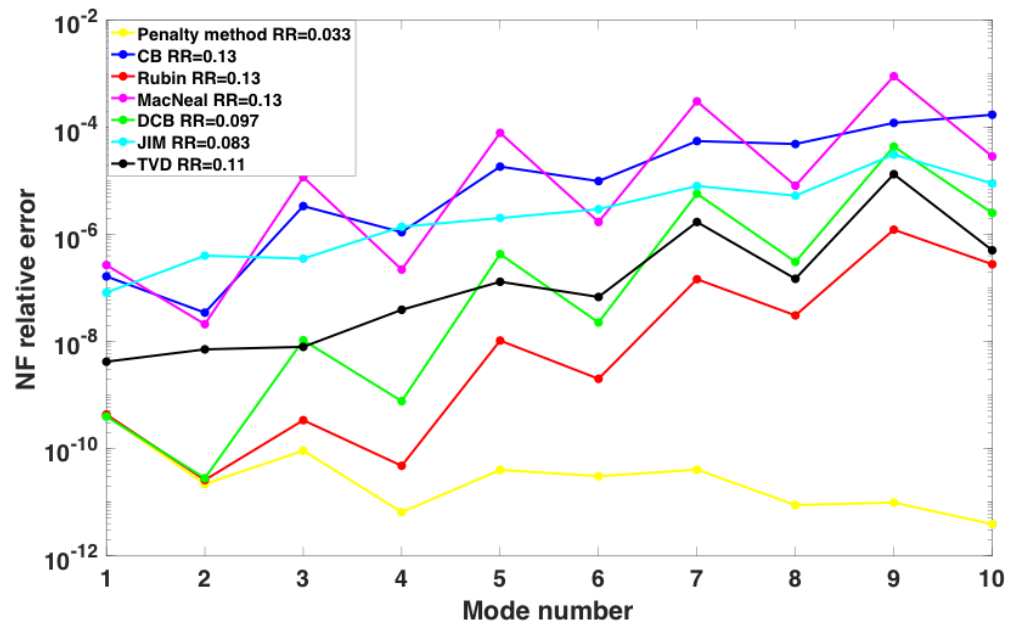

Fig. 5 NF relative errors of the jointed beams between different ROM methods (RR:reduction ratio)

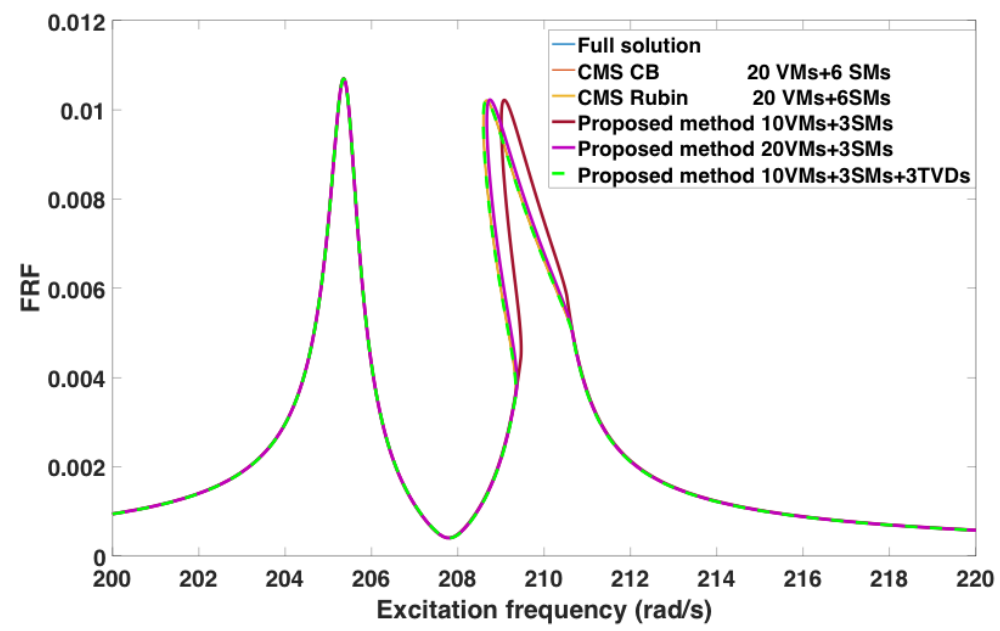

Fig. 6 Forced Frequency response comparisons between CB,Rubin and Penalty ROM methods 
For nonlinear analysis, the linear springs on the contact interface are replaced by using Jenkin contact friction model. Fig. 5 shows the comparison of the frequency response functions (FRFs) between the proposed method and Rubin, CB methods. The structure is excited in the middle of the first substructure in the $y$ direction. The two structure would be separated if the excitation level was large, which would activate the soften effect of the in-phase and out-of-phase bending modes. For Rubin and $\mathrm{CB}$ methods, the number of nominal modes is 10 while the number of static modes is equal to the number of non-linear DOFs in the joint. Fig. 6 shows the peak of the out-of-phase resonance shafts on the left but the amplitude of the response remain unchanged. It means that the jointed structure experiences the separation in resonance frequency region. CB and Rubin have the same FRFs as that from the full solution. Using 10 vibrational modes and 3 static modes, the penalty approach leads to the noticeable errors. When increasing the number of vibration modes to 20 , the accuracy with the proposed method improves but one still can observe the clear discrepancy. This is because the introduced non-linear force on the contact interface would affect the linear reduced basis and cause mode interaction between them [11]. This mean the linear reduced basis need to be calibrated in order to accurately represent the dynamics of coupled systems. The TVD method is one of the effective approaches for the calibration when this coupling between linear vibration modes is significant[11]. Three TVDs vectors are then added into the reduced basis to assess how this would improve the accuracy of the propose method. The results show that the proposed method with TVDs can obtain the same FRF as that from the full solution. Comparing to the CB and Rubin methods, the size of this reduced jointed structure model using the proposed method can be reduced by $40 \%$ even near the resonance frequency region.

\section{Conclusions and future work}

A novel penalty-based reduced order modelling approach has been proposed for dynamic analysis of a jointed structure with localised contact friction non-linearities. The formulation of the proposed approach has been presented where the contact friction is modelled by a Jenkins model. We also showed how the proposed method can be effectively integrated with the harmonic balanced method, AFT and nonlinear solver. A case case using jointed beam has been carried out to demonstrate the proposed method. The result obtained from the penalty approach was compared with the full solution and also classical Rubin and CB methods. The initial results show the method can effectively capture the FRFs of the non-linear dynamic system. TVDs are particularly useful to improve the accuracy when the contact interface is largely in a non-linear condition. Comparing to the classical CMS methods, the proposed method can reduce the size of ROM further when most of contact nodes are in a stuck condition.

The main objective of this paper was to present the formulation of this new reduced order modelling approach for a joint structure, and demonstrate the concept 
with a simple case study. Further developments would be needed to couple the proposed method with continuation techniques for HBM. This would enable the automatic size updating during the forced frequency response simulations. Also, high

fidelity models are needed to further test and validate the proposed method and effects of TVDs on the dynamics of joint structures.

Acknowledgements The authors would like to acknowledge the support of Rolls-Royce plc for this research through the Vibration University Technology Centre (UTC) at the Imperial College London, UK. Special acknowledgement goes also to GEMiniDS WP3 - Innovate UK Project 113088 , which is jointly supported by Innovate UK and Rolls- Royce plc.

\section{References}

[1] Bograd, S., Reuss, P., Schmidt, A., Gaul, L., And Mayer, M. Modeling the dynamics of mechanical joints. Mechanical Systems and Signal Processing 25, 8 (2011), 2801-2826.

[2] CRAig, R., AND BAmpton, M. Coupling of substructures for dynamic analyses. AIAA journal 6, 7 (1968), 1313-1319.

[3] GRUBER, F. M., AND RIXEN, D. J. Evaluation of substructure reduction techniques with fixed and free interfaces. Strojniški vestnik-Journal of Mechanical Engineering 62, 7-8 (2016), 452-462.

[4] Krack, M., Salles, L., And Thouverez, F. Vibration prediction of bladed disks coupled by friction joints. Archives of Computational Methods in Engineering 24, 3 (Jul 2017), 589-636.

[5] Pesaresi, L., SAlles, L., Jones, A., Green, J., And Schwingshackl, C. Modelling the nonlinear behaviour of an underplatform damper test rig for turbine applications. Mechanical Systems and Signal Processing 85 (2017), $662-679$.

[6] Petrov, E. A high-accuracy model reduction for analysis of nonlinear vibrations in structures with contact interfaces. Journal of Engineering for Gas Turbines and Power 133, 10 (2011), 102503.

[7] PinnaU, R. Model reduction via proper orthogonal decomposition. In Model Order Reduction: Theory, Research Aspects and Applications. Springer, 2008, pp. 95-109.

[8] RuBin, S. Improved component-mode representation for structural dynamic analysis. AIAA journal 13, 8 (1975), 995-1006.

[9] Sarrouy, E., AND SinOU, J.-J. Non-linear periodic and quasi-periodic vibrations in mechanical systems-on the use of the harmonic balance methods. In Advances in Vibration Analysis Research. InTech, 2011.

[10] WitteVeEn, W., AND IRSCHIK, H. Efficient mode based computational approach for jointed structures: joint interface modes. AIAA Journal 47, 1 (2009), 252-263. 
[11] Witteveen, W., AND PichleR, F. Efficient model order reduction for the dynamics of nonlinear multilayer sheet structures with trial vector derivatives. Shock and Vibration 2014 (2014).

[12] Yuan, J., El-Haddad, F., SAlles, L., AND Wong, C. Numerical assessment of reduced order modeling techniques for dynamic analysis of jointed structures with contact nonlinearities. Journal of Engineering for Gas Turbines and Power 141, 3 (2019), 031027.

[13] Yuan, J., Scarpa, F., Allegri, G., Titurus, B., Patsias, S., and RaJASEKARAN, R. Efficient computational techniques for mistuning analysis of bladed discs: A review. Mechanical Systems and Signal Processing 87 (2017), $71-90$. 